\title{
OPEN Validation for measurements of skeletal muscle areas using low-dose chest computed tomography
}

\author{
Woo Hyeon Lim ${ }^{1,2}$ \& Chang Min Park $2,3,4,5 \square$
}

Various methods were suggested to measure skeletal muscle areas (SMAs) using chest low-dose computed tomography (chest LDCT) as a substitute for SMA at 3rd lumbar vertebra level (L3-SMA). In this study, four SMAs (L1-SMA, T12-erector spinae muscle areas, chest wall muscle area at carina level, pectoralis muscle area at aortic arch level) were segmented semi-automatically in 780 individuals taking concurrent chest and abdomen LDCT for healthcare screening. Four SMAs were compared to L3-SMA and annual changes were calculated from individuals with multiple examinations $(n=101)$. Skeletal muscle index (SMI; SMA/height ${ }^{2}$ ) cut-off for sarcopenia was determined by lower 5 th percentile of young individuals (age $\leq 40$ years). L1-SMA showed the greatest correlation to L3-SMA (men, $\mathrm{R}^{2}=0.7920$; women, $\left.\mathrm{R}^{2}=0.7396\right)$, and the smallest annual changes $(0.3300 \pm 4.7365 \%)$ among four SMAs. L1-SMI cut-offs for determining sarcopenia were $39.2 \mathrm{~cm}^{2} / \mathrm{m}^{2}$ in men, and $27.5 \mathrm{~cm}^{2} / \mathrm{m}^{2}$ in women. Forty-six men (9.5\%) and ten women (3.4\%) were found to have sarcopenia using L1-SMI cut-offs. In conclusion, L1-SMA could be a reasonable substitute for L3-SMA in chest LDCT. Suggested L1-SMI cut-offs for sarcopenia were $39.2 \mathrm{~cm}^{2} / \mathrm{m}^{2}$ for men and $27.5 \mathrm{~cm}^{2} / \mathrm{m}^{2}$ for women in Asian.

Sarcopenia, characterized by decreased in muscle strength, quantity, and quality ${ }^{1}$, is garnering attention in medical fields including oncology $y^{2-4}$, pulmonology $y^{5}$, and cardiology $y^{6}$. Older adults are vulnerable to sarcopenia as well ${ }^{7}$, and they have often undergone chest low-dose computed tomography (LDCT) for lung cancer screening ${ }^{8}$, and diagnosis and monitoring for other various pulmonary pathologies including lung cancer, interstitial lung diseases (ILD) ${ }^{9}$, and chronic obstructive pulmonary disease (COPD) ${ }^{10}$. Unfortunately, however, typical chest LDCT does not cover 3rd lumbar vertebra (L3) level, which is considered as a reference standard for sarcopenia diagnosis for body imaging-based study ${ }^{2-6}$.

Thus, investigators tried to utilize various anatomic landmarks as substitutes for skeletal muscle area (SMA) at L3 level (L3-SMA) in chest CT: (1) SMA at 1st lumbar vertebral body (L1) level (L1-SMA) ${ }^{11-13}$, (2) erector spinae muscle area (ESMA) at 12th thoracic vertebral body (T12) level (T12-ESMA) $)^{14-16}$, (3) chest wall muscle area (CWMA) at carina (C-CWMA) ${ }^{17}$ or 4 th thoracic vertebral body (T4) level ${ }^{15}$ where carina is usually located, and (4) pectoralis muscle area (PMA) just above aortic arch level (AA-PMA) ${ }^{11,13,14,18}$.

However, comprehensive comparison between L3-SMA and multiple SMAs previously suggested as substitutes for L3-SMA using chest CT does not exist so far. Through this comparison, researchers could obtain more objective information on each SMA and standardize the utilization of chest CT in sarcopenia study. Furthermore, additional information such as race-specific cut-offs for each SMA in chest LDCT or whether the skeletal muscles decreased evenly according to their location could be obtained in general population-based study ${ }^{19}$.

Through this study, it is firstly aimed to suggest the optimal substitution upon chest LDCT examinations for L3-SMA and present Asian specific cut-offs for sarcopenia diagnosis. Second, factors related to uneven decrease of skeletal muscles, if so, were analyzed.

\footnotetext{
${ }^{1}$ Department of Radiology, Namwon Medical Center, Namwon-si, Jeollabuk-do, Korea. ${ }^{2}$ Department of Radiology, Seoul National University College of Medicine, 101 Daehak-ro, Chongno-gu, Seoul 03080, Korea. ${ }^{3}$ Institute of Radiation Medicine, Seoul National University Medical Research Center, Seoul, Korea. ${ }^{4}$ Cancer Research Institute, Seoul National University College of Medicine, Seoul, Korea. ${ }^{5}$ Institute of Medical and Biological Engineering, Medical Research Center, Seoul National University, Seoul, Korea. ${ }^{\circledR}$ email: cmpark.morphius@gmail.com
} 


\begin{tabular}{|l|l|l|}
\hline \multirow{2}{*}{ Age (years) } & Men $(\mathbf{n}=482)$ & Women $(\mathbf{n}=298)$ \\
\hline & $50.9 \pm 10.5$ & $51.4 \pm 10.8$ \\
\cline { 2 - 3 } & $($ median, 51.0) & (median, 52.0) \\
\cline { 2 - 3 } & $($ range, 23.0-77.0) & $($ range, 21.0-79.0) \\
\hline Body weight $(\mathrm{kg})$ & $72.2[65.3,79.9]$ & $57.9[52.5,63.8]$ \\
\hline Height $(\mathrm{cm})$ & $169.7 \pm 6.1$ & $157.3 \pm 6.1$ \\
\hline Body-mass index $\left(\mathrm{kg} / \mathrm{m}^{2}\right)$ & $25.2[23.2,27.3]$ & $23.3[21.7,25.7]$ \\
\hline Waist circumference $(\mathrm{cm})^{\star}$ & $84.0[79.0,89.0]$ & $75.0[69.8,80.3]$ \\
\hline Abdominal obesity* & $104(21.7 \%)$ & $45(15.2 \%)$ \\
\hline Smoking & \multicolumn{2}{|l}{} \\
\hline Never smoker & $265(55.0 \%)$ & $294(98.7 \%)$ \\
\hline Ex-smoker & $19(3.9 \%)$ & $1(0.3 \%)$ \\
\hline Current smoker & $198(41.1 \%)$ & $3(1.0 \%)$ \\
\hline Underlying disease & \multicolumn{2}{|l}{} \\
\hline Hypertension & $113(23.4 \%)$ & $48(16.1 \%)$ \\
\hline Diabetes mellitus & $63(13.1 \%)$ & $22(7.4 \%)$ \\
\hline Dyslipidemia & $87(18.0 \%)$ & $47(15.8 \%)$ \\
\hline
\end{tabular}

Table 1. Information on 780 individuals taking healthcare screening examination. Numerical variables with normal distribution were provided with mean \pm standard deviation, while those not showing normal distribution were provided with median [interquartile range]. Abdominal obesity was defined as a waist circumference $\geq 90 \mathrm{~cm}$ in men, and $\geq 85 \mathrm{~cm}$ in women. ${ }^{\star}$ These values were extracted from 776 individuals consisting of 479 men and 297 women.

\section{Results}

Table 1 summarizes demographics, anthropometric information and clinical information of 780 individuals included in this study.

Comparison of chest LDCT-derived muscle areas with L3-SMA. Among four chest CT-derived muscle areas, L1-SMA (men, $\mathrm{R}^{2}=0.7920$; women, $\mathrm{R}^{2}=0.7396$ ) showed the greatest correlation to L3-SMA, while AA-PMA (men, $\mathrm{R}^{2}=0.3052$; women, $\mathrm{R}^{2}=0.2672$ ) exhibited the least correlation to L3-SMA (Fig. 1). L1-SMA showed the least annual changes and standard variation $(0.3300 \pm 4.7365 \%)$ among four areas (Fig. 2). The T12ESMA and C-CWMA were influenced by reconstruction methods $(P<0.05)$.

Chest LDCT-based definition of sarcopenia. Trends of all SMAs and SMIs according to age were described in Supplementary Fig. 1. The cut-off for L1-skeketal muscle index (L1-SMI) for sarcopenia was $39.2 \mathrm{~cm}^{2} / \mathrm{m}^{2}$ for men, and $27.5 \mathrm{~cm}^{2} / \mathrm{m}^{2}$ for women, respectively (Table 2). With this cut-off, $46 \mathrm{men}(9.5 \%)$ and 10 women $(3.4 \%)$ were found to have sarcopenia. Agreements to L3-SMI for defining sarcopenia were substantial for L1-SMI $(\kappa=0.622)$, moderate for C-CWMI $(\kappa=0.550)$ and AA-PMI $(\kappa=0.439)$, while T12-ESMI showed fair agreement $(\kappa=0.361)$ (Table 2$)$.

Risk factors for sarcopenia based on chest LDCT. In univariate logistic regression, sex, body weight, height, body-mass index (BMI), smoking status and factors related to central obesity (waist circumference [WC], visceral fat area [VFA], subcutaneous fat area [SFA], total fat area [TFA]) were found to be associated with sarcopenia based on L1-SMI (Table 3). In multivariate logistic regression, sarcopenia based on L1-SMI was significantly related with men (adjusted odds ratio $[\mathrm{OR}]=25.856$, 95th percentile confidence interval [95\% $\mathrm{CI}]=8.714$ to $76.720, P<0.001)$, lower $\mathrm{BMI}(\mathrm{OR}=0.336,95 \% \mathrm{CI}=0.251$ to $0.449, P<0.001)$, and greater VFA $(\mathrm{OR}=1.013,95 \% \mathrm{CI}=1.001$ to $1.025, P=0.030)$. When VFA was replaced by SFA or TFA, men, lower BMI, and central obesity were persistently related with sarcopenia $(P<0.05)$.

Factors related to accelerated decrease of chest wall muscle areas. The lower C-CWMA/L1-SMA ratio $(\mathrm{C} / \mathrm{L} 1$ ratio) was significantly associated with older age $(P<0.001)$ (Fig. 3$)$, women $(P=0.007)$, and greater BMI $(P<0.001)$, while L3-SMI was not the factors related with $\mathrm{C} / \mathrm{L} 1$ ratio. In addition, current smokers showed lower $\mathrm{C} / \mathrm{L} 1$ ratio compared to never smokers after adjustment of age, sex, and BMI $(P=0.042)$, and this relationship was dose-dependent in current smokers with exact pack-year (PY) $(n=137, P=0.012)$ (Fig. 3). Smoking status was not associated with L1-SMI after adjustment of age, sex, BMI, and VFA.

\section{Discussion}

In this study, four representative anatomic landmarks were selected (L1-SMA, T12-ESMA, C-CWMA, and AAPMA), because those were well validated in several studies ${ }^{11-18}$. The T12-SMA ${ }^{20}$ was not evaluated in this study, because T12-SMA which was smaller than L1-SMA, showed significant correlation with L1-SMA ${ }^{21}$. Besides, the utilization of intercostal muscles alone ${ }^{22}$ was not considered because their small areas could be vulnerable to measurement errors. Among four regions of interest, L1-SMA showed the greatest correlation to L3-SMA 
(a)

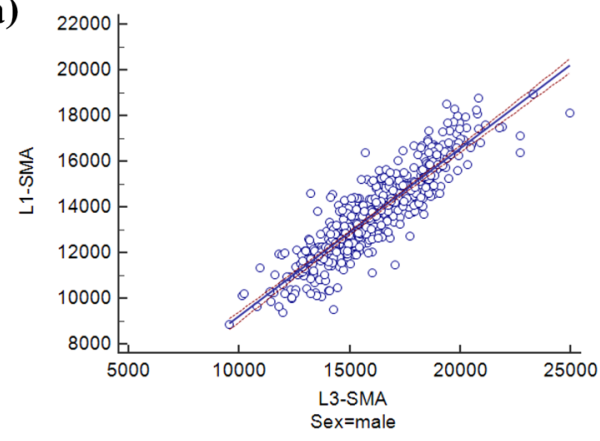

(b)

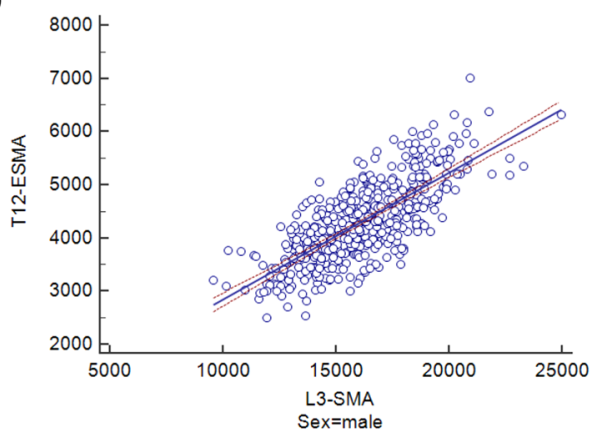

(c)

(d)
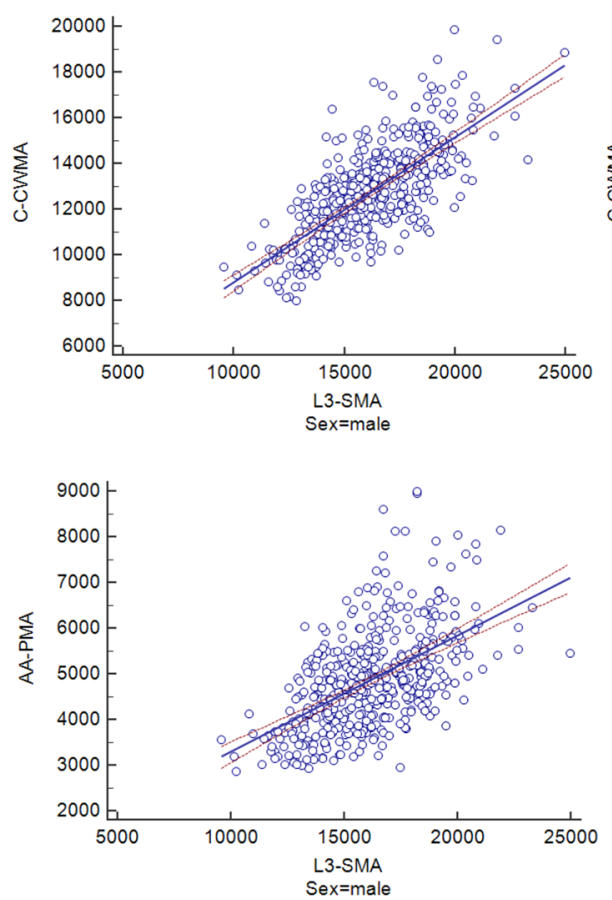
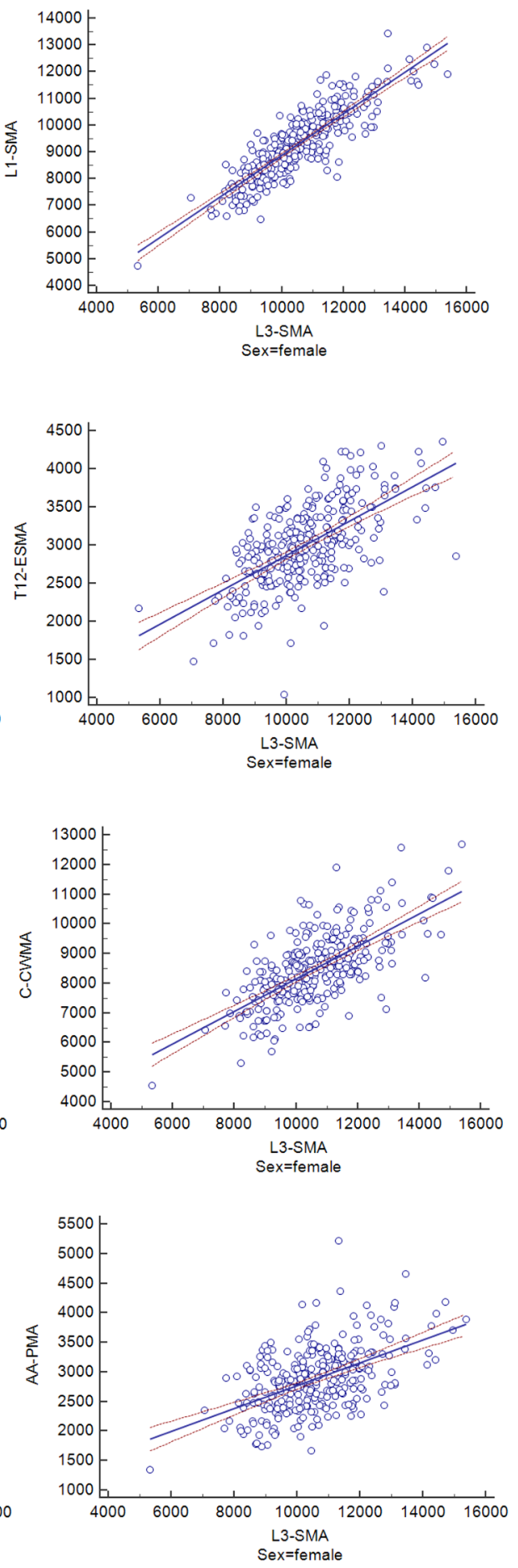

Figure 1. Correlation of skeletal muscle area (SMA) between L3-SMA and variable anatomic landmarks in chest CT: (a) L1-SMA (men, $\mathrm{R}^{2}=0.7920$; women, $\mathrm{R}^{2}=0.7396$ ), (b) T12-ESMA (men, $\mathrm{R}^{2}=0.5557$; women, $\mathrm{R}^{2}=0.3834$ ), (c) C-CWMA (men, $\mathrm{R}^{2}=0.5306$; women, $\mathrm{R}^{2}=0.4427$ ), and d) AA-PMA (men, $\mathrm{R}^{2}=0.3052$; women, $\left.\mathrm{R}^{2}=0.2672\right)$.

regardless of sex, while annual change was the smallest. In addition, in defining sarcopenia using region-specific cut-offs, L1-SMI provided the greatest agreement with L3-SMI. These findings suggested that L1-SMA could be a reasonable substitute for L3-SMA in chest CT-based study.

In this study, the importance of cut-offs to define sarcopenia in imaging-based study was also reaffirmed. One of the most commonly used cut-offs was those suggested by Prado et al. (men, $55.0 \mathrm{~cm}^{2} / \mathrm{m}^{2}$; women, $39.0 \mathrm{~cm}^{2} /$ $\mathrm{m}^{2}$ ), which focused on prognostication in oncologic patients ${ }^{2}$. Although the prediction of prognosis in a specific 


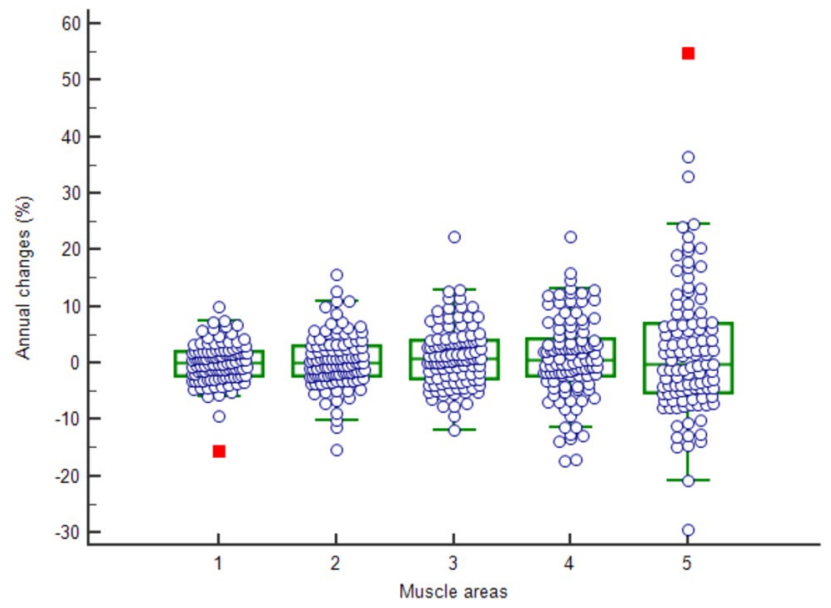

Figure 2. Box-and-Whisker plots regarding annual changes (\%) of variable muscle areas ([1] skeletal muscle area at 3rd lumbar vertebra level, $0.0725 \pm 3.5308 \%$; [2] skeletal muscle area at 1 st lumbar vertebra level, $0.3300 \pm 4.7365 \%$; [3] = erector spinae muscle area at 12 th thoracic vertebra level, $1.1614 \pm 5.3488 \%$; [4] chest wall muscle area at carina level, $0.8622 \pm 7.1192 \%$ [5]; pectoralis muscle area just above aortic arch level, $2.0202 \pm 11.8910 \%)$.

\begin{tabular}{|l|l|l|l|}
\hline & Men & Women & Sarcopenia (n) \\
\hline L3-SMA $\left(\mathrm{cm}^{2}\right) / \mathrm{SMI}\left(\mathrm{cm}^{2} / \mathrm{m}^{2}\right)$ & $142.1 / 46.3$ & $85.6 / 32.8$ & $55(7.1 \% ; \mathrm{M}: \mathrm{W}=48: 7)$ \\
\hline L1-SMA $\left(\mathrm{cm}^{2}\right) / \mathrm{SMI}\left(\mathrm{cm}^{2} / \mathrm{m}^{2}\right)$ & $116.2 / 39.2$ & $73.7 / 27.5$ & $56(7.2 \% ; \mathrm{M}: \mathrm{W}=46: 10)$ \\
\hline T12-ESMA $\left(\mathrm{cm}^{2}\right) /$ ESMI $\left(\mathrm{cm}^{2} / \mathrm{m}^{2}\right)$ & $34.3 / 11.6$ & $22.9 / 8.4$ & $49(6.3 \% ; \mathrm{M}: \mathrm{W}=39: 10)$ \\
\hline C-CWMA $\left(\mathrm{cm}^{2}\right) / \mathrm{CWMI}\left(\mathrm{cm}^{2} / \mathrm{m}^{2}\right)$ & $114.2 / 36.2$ & $74.1 / 26.6$ & $68(8.7 \% ; \mathrm{M}: \mathrm{W}=53: 15)$ \\
\hline AA-PMA $\left(\mathrm{cm}^{2}\right) /$ PMI $\left(\mathrm{cm}^{2} / \mathrm{m}^{2}\right)$ & $40.2 / 13.2$ & $19.2 / 6.9$ & $77(9.9 \% ; \mathrm{M}: \mathrm{W}=72: 5)$ \\
\hline
\end{tabular}

Table 2. The cut-offs to define sarcopenia in Asian population. SMA = skeletal muscle area (L3=at 3rd lumbar vertebra level, $\mathrm{L} 1=$ at 1 st lumbar vertebra level), SMI = skeletal muscle index $(\mathrm{L} 3=$ at $3 \mathrm{rd}$ lumbar vertebra level, $\mathrm{L} 1=$ at 1 st lumbar vertebra level), T12-ESMA = erector spinae muscle area at 12th thoracic vertebra level, T12$\mathrm{ESMI}=$ erector spinae muscle index at 12th thoracic vertebra level, $\mathrm{C}$-CWMA $=$ chest wall muscle area at carina level, $\mathrm{C}-\mathrm{CWMI}=$ chest wall muscle index at carina level, AA-PMA = pectoralis muscle area just above aortic arch level, AA-PMI = pectoralis muscle index just above aortic arch level, $\mathrm{M}=\mathrm{men}, \mathrm{W}=$ women.

disease is important, to understand age-dependent changes and diagnose sarcopenia in general population, the cut-offs of sarcopenia should be determined based on healthy general population ${ }^{21}$, which is similar to T-score in osteoporosis.

Kim et al. suggested L1-SMI cut-offs (men, $46.0 \mathrm{~cm}^{2} / \mathrm{m}^{2}$; women, $29.0 \mathrm{~cm}^{2} / \mathrm{m}^{2}$ ), which were calculated from linear regression between L3-SMI and L1-SMI ${ }^{13}$. Because Kim et $\mathrm{al}^{13}$ used predefined L3-SMI cut-offs from Prado et $\mathrm{al}^{2}$, these L1-SMI cut-offs were quite greater than ours (men, $39.2 \mathrm{~cm}^{2} / \mathrm{m}^{2}$; women, $27.5 \mathrm{~cm}^{2} / \mathrm{m}^{2}$ ). L1-SMI cutoffs from our study also showed differences from those based on healthy American populations (men, $34.6 \mathrm{~cm}^{2} /$ $\mathrm{m}^{2}$; women, $\left.25.9 \mathrm{~cm}^{2} / \mathrm{m}^{2}\right)^{21}$. It might be necessary to explore which L1-SMI cut-off reflects skeletal muscle mass more precisely by comparing the L1-SMI cut-off with grip strength and lean body mass ${ }^{23}$.

Our study demonstrated the accelerated decrease of C-CWMA compared to L1-SMA with aging. One of the notable finding is that C/L1 ratio was not associated with L3-SMI. The accelerated decrease of C-CWMA is rarely reported so far. It could be possible that L3-SMA and L1-SMA were likely to be associated with general medical condition, while C-CWMA would be more likely to be affected by other factors such as morphology of thoracic cage ${ }^{24}$ on top of the general medical condition.

In addition, current smoking seemed to have different effects on decrease of SMA according to the location. Current smoking was associated with accelerated decrease of C-CWMA, while L1-SMI itself was not associated with smoking status. Diaz et $\mathrm{al}^{25}$ demonstrated the low muscle mass in chest CT was related to increased mortality in current smokers. Cigarette smoking is a known risk factor for skeletal muscle loss, by inducing proteolysis and inhibiting protein synthesis ${ }^{26}$. However, it is unclear how smoking contributes accelerated decrease of C-CWMA compared to L1-SMA, and causal relationship between smoking and accelerated decrease of C-CWMA could not be determined solely based on our results. Because patients with COPD showed tendency toward having weakened chest wall muscles ${ }^{27}$, one of the possible explanation is that subclinical airflow limitation could also result in decrease of chest wall muscles in current smokers. In this regard, further studies investigating the relationship between smoking, airflow limitation and chest wall muscle should be needed. 


\begin{tabular}{|c|c|c|c|c|}
\hline Variables & Sarcopenia $(+)(n=56)$ & Sarcopenia $(-)(n=724)$ & Odds ratio (95th C.I) & $p$-value \\
\hline Age (years) & $52.5 \pm 9.5$ & $51.0 \pm 10.6$ & $1.014(0.988,1.040)$ & 0.309 \\
\hline $\operatorname{Sex}(M: W)(n)$ & $46: 10$ & $436: 288$ & $3.039(1.509,6.118)$ & 0.002 \\
\hline Body weight (kg) & $59.8[54.0,64.5]$ & $67.6[59.4,76.4]$ & $0.925(0.900,0.951)$ & $<0.001$ \\
\hline Height $(\mathrm{cm})$ & $168.3[163.9,173.7]$ & $165.3[158.5,171.3]$ & $1.061(1.026,1.098)$ & $<0.001$ \\
\hline BMI $\left(\mathrm{kg} / \mathrm{m}^{2}\right)$ & $20.7[19.6,21.8]$ & $24.8[22.8,27.1]$ & $0.542(0.468,0.627)$ & $<0.001$ \\
\hline \multicolumn{5}{|l|}{ Smoking } \\
\hline Smoking status (n) & & & & 0.01 \\
\hline Current vs. Never & $23: 30$ & $178: 529$ & $2.279(1.290,4.026)$ & 0.005 \\
\hline Ex vs. Never & $3: 30$ & $17: 529$ & $3.119(0.864,11.207)$ & 0.083 \\
\hline $\mathrm{PY}(\mathrm{n}=696 ; 46: 650)$ & $7.9 \pm 12.8$ & $4.1 \pm 10.2$ & $1.027(1.004,1.050)$ & 0.02 \\
\hline Hypertension (n) & 7 & 154 & $0.529(0.235,1.191)$ & 0.124 \\
\hline Diabetes mellitus (n) & 6 & 79 & $0.980(0.407,2.358)$ & 0.964 \\
\hline Dyslipidemia (n) & 5 & 129 & $0.452(0.177,1.155)$ & 0.097 \\
\hline \multicolumn{5}{|l|}{ Central obesity ${ }^{\star}$} \\
\hline $\mathrm{WC}(\mathrm{cm})$ & $73.7 \pm 6.9$ & $81.3 \pm 8.9$ & $0.899(0.867,0.932)$ & $<0.001$ \\
\hline VFA $\left(\mathrm{cm}^{2}\right)$ & $59.5[37.0,97.4]$ & $105.7[66.7,143.8]$ & $0.983(0.977,0.990)$ & $<0.001$ \\
\hline $\mathrm{SFA}\left(\mathrm{cm}^{2}\right)$ & $92.4[61.5,125.1]$ & $150.6[119.1,192.3]$ & $0.973(0.965,0.980)$ & $<0.001$ \\
\hline TFA $\left(\mathrm{cm}^{2}\right)$ & $162.2 \pm 67.3$ & $266.0 \pm 91.4$ & $0.986(0.982,0.990)$ & $<0.001$ \\
\hline
\end{tabular}

Table 3. Univariate logistic regression analyses for evaluating factors related with sarcopenia defined by skeletal muscle index at 1st lumbar vertebra level (L1-SMI). Numerical variables with normal distribution were provided with mean \pm standard deviation, while those not showing normal distribution were provided with median [interquartile range] except pack-year. $\mathrm{M}=$ men, $\mathrm{W}=$ women, $\mathrm{n}=$ number, $\mathrm{BMI}=$ body-mass index, $\mathrm{PY}=$ Pack-Year, $\mathrm{WC}=$ waist circumference, $\mathrm{FA}=$ fat area (V:visceral, S:subcutaneous, T:total). ${ }^{*}$ Numbers of evaluated individuals (WC, $\mathrm{n}=776,54: 722$; VFA, $\mathrm{n}=779,55: 724$; SFA, $\mathrm{n}=739,55: 684$; TFA, $\mathrm{n}=739,55: 684$ ).

In this study, the SMA and SMI of men generally tended to decrease with aging, while this relationship was not confirmed in women. According to results from Magudia et $\mathrm{al}^{19}$, age-independence of SMA in women seems not to be obvious in White non-Hispanic and Black. One of the possible explanation for this finding is that ageindependence of SMA in women is a result of the difference in lifestyle (such as exercise, diet), which could not be assessed objectively on this retrospective study. Multicenter studies should be needed to investigate whether this finding is Asian characteristic or originates from the difference of lifestyle.

In our study, image reconstruction methods have impact on T12-ESMA and C-CWMA. However, impact of image reconstruction methods was not the main focus of our study, because the magnitude of influence of image reconstruction methods on SMA measurement is not substantial ${ }^{28}$. Recently, deep learning-based body composition segmentation at CT images was validated ${ }^{29-31}$ and this technique could provide robust segmentation from noise ${ }^{32}$. In addition, deep learning also enables researchers to perform time-consuming process more easily ${ }^{33}$. We believe that utilization of deep learning-based technique can bring another breakthrough to body composition measurements.

There were several limitations of this study. First, this was single institutional retrospective study, and only Asian individuals were included in this study. Thus, there could be of unknown bias and the generalization of our results could be limited. The usage of contrast-enhanced CT images might have impact on segmentation of muscle, although small differences of SMI were observed after contrast administration ${ }^{34}$. However, contrast enhancement provided better discrimination of abdominal solid organs, and this was helpful in segmenting skeletal muscles semi-automatically using abdomen CT. Finally, age-dependent changes and the different effect of smoking on each SMA were not fully understood based on this study, because such findings were not correlated with physiologic changes. Further studies to elucidate the relationship of selective muscle atrophy with aging and smoking should be needed in terms of physiologic changes.

In conclusion, L1-SMA could be a reasonable substitute for L3-SMA in chest CT-based study for sarcopenia and suggested cut-offs for sarcopenia using L1-SMI were $39.2 \mathrm{~cm}^{2} / \mathrm{m}^{2}$ for men and $27.5 \mathrm{~cm}^{2} / \mathrm{m}^{2}$ for women in Asian. Current smoking significantly contributed to accelerated decrease of C-CWMA in general population.

\section{Methods}

This retrospective study was approved by Public Institutional Review Board designated by Ministry of Health and Welfare, Republic of Korea (approval number: P01-202,011-21-015) with a waiver of informed consent, and all experiments were performed in accordance with relevant guidelines and regulations.

Study population. From January 2017 to December 2019, 918 pairs of contrast-enhanced chest and abdomen LDCT on the same day for healthcare screening were identified in a secondary-referral hospital. In South Korea, there are various types of healthcare screening programs depending on institutes and those who receive these programs are either supported by their companies and local governmental organizations or are examined voluntarily $^{35,36}$. Among them, 29 pairs of examinations were excluded from this study because of following rea- 
(a)

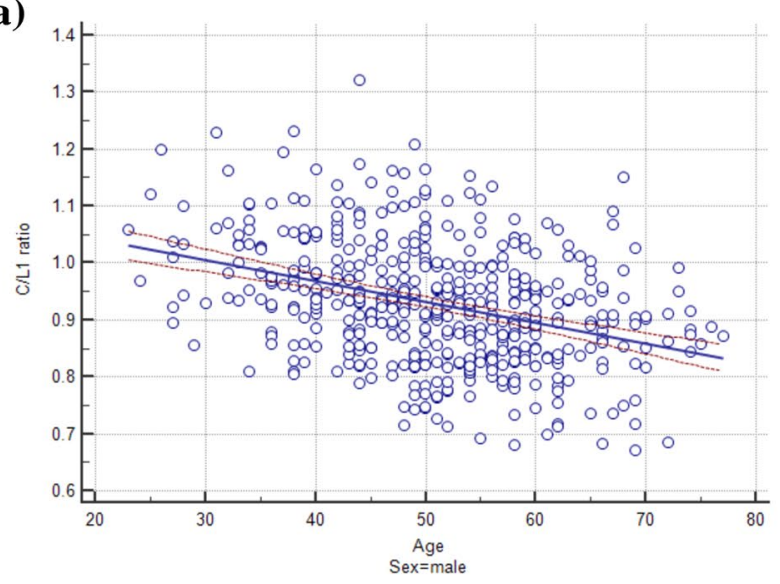

(b)

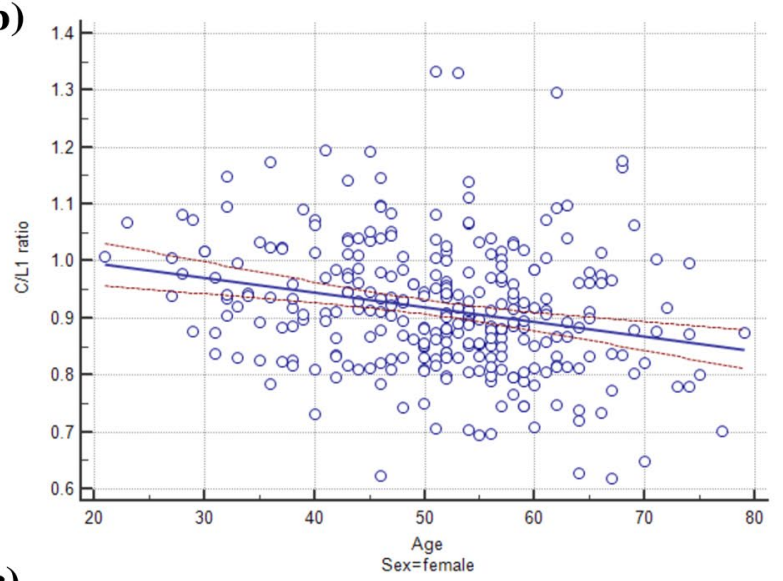

(c)

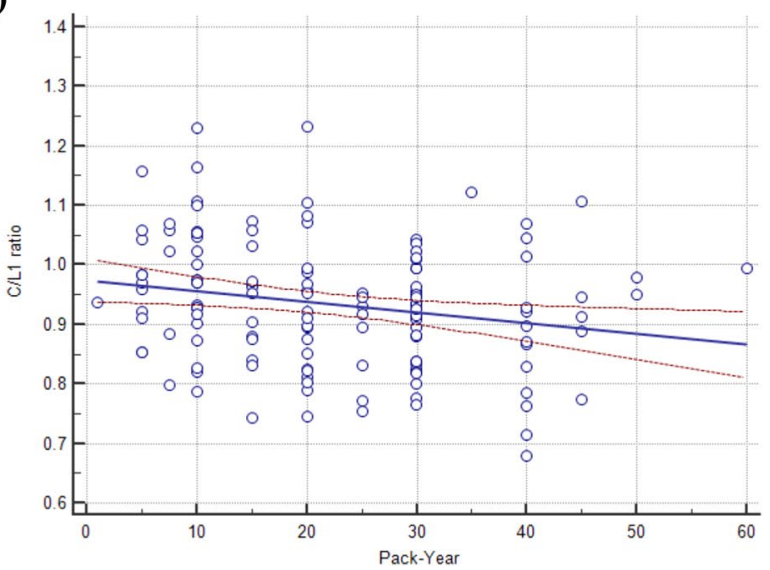

Figure 3. The $\mathrm{C} / \mathrm{L} 1$ ratio (the ratio of chest wall muscle area at carina and skeletal muscle area at 1 st lumbar vertebral level) according to age and smoking status: $(\mathbf{a}, \mathbf{b})$ The $\mathrm{C} / \mathrm{L} 1 \mathrm{ratio}$ decreased with age $(P<0.001)$ in general population, and $(\mathbf{c})$ pack-year $(P=0.012)$ in current smokers.

sons: 1) patients with history of cancer, which could affect the skeletal muscle mass $(\mathrm{n}=18), 2)$ patients with inappropriate arm posture or incomplete scan coverage (i.e. muscles of interest were not fully included) $(n=4)$, 3) deformity in muscles of interest (i.e. patients who received augmentation mammoplasty) $(\mathrm{n}=2)$, 4) patients who received spine surgery that caused significant beam-hardening artifact at areas of interest $(n=3)$, and 5) lack of height data $(\mathrm{n}=2)$.

Finally, 780 individuals (mean age, $50.9 \pm 10.5$ years in men and $51.4 \pm 10.8$ years in women) with 889 pairs of examinations were included for this study. Among them, 101 individuals received multiple examinations during study period (two CT examinations for 93 individuals and three examinations for 8). Data from multiple examinations were only used to explore annual change rates, and data of the first examination were only used for statistical analysis in individuals with multiple examinations. 
CT protocol, measurement of SMAs and fat area. CT imaging was performed using SOMATOM Definition AS + (Siemens, Erlangen, Germany). Chest and abdomen LDCT were scanned with 50mAs/100kVp and $160 \mathrm{mAs} / 100 \mathrm{kVp}$, respectively, after contrast media administration. To minimize the difference according to contrast-media usage, both contrast-enhanced chest and abdomen LDCT were utilized in this study. For skeletal muscle segmentation, chest CT images with lesser noise were used. During study period, three different image reconstruction methods were applied: 1) $5 \mathrm{~mm}$ slice-thickness with I50f. filter $(\mathrm{n}=353), 2) 5 \mathrm{~mm}$ slice-thickness with I30f. filter $(\mathrm{n}=329)$, and 3) $3 \mathrm{~mm}$ slice-thickness with I30f. filter $(\mathrm{n}=98)$. All abdomen CT images were reconstructed with $5 \mathrm{~mm}$ slice-thickness with I30f.

Each skeletal muscle was segmented semi-automatically by using open software (3D Slicer Chest Imaging Platform, version 4.10.2), and Hounsfield units of -29 to 150 were considered skeletal muscles ${ }^{2}$. The L3-SMA ${ }^{2-6}$, L1-SMA $^{11-13}$, T12-ESMA ${ }^{14-16}$, C-CWMA ${ }^{17}$ and AA-PMA ${ }^{11,13,14,18}$ were segmented for this study (Supplementary Fig. 2). For segmentation of C-CWMA and AA-PMA, chest CT images were used. In cases that T12 or L1 level was not covered in chest CT, segmentation of T12-ESMA or L1-SMA was done by using abdomen CT images. Because chest CT images with I50f filter showed significant noise at thoracoabdominal junction (i.e. T12 and L1 levels), segmentation of T12-ESMA and L1-SMA were performed with abdomen CT images when available, instead of using chest CT images (Supplementary Fig. 3). The C/L1 ratio was calculated to assess the uneven decrease of skeletal muscles according to their locations. Among three thoracic skeletal muscle areas (AA-PMA, C-CWMA, and T12-ESMA), C-CWMA was selected because of its low annual variability (Fig. 2) and for comprehensive evaluation of thoracic skeletal muscles.

Sarcopenia was defined by using lower 5 th percentile values of SMI (SMA/height $\left.{ }^{2}\right)^{2}$ from young individuals (age $\leq 40$ years) $^{21}$. To explore the effect of central obesity on skeletal muscle mass, VFA at umbilicus level was also segmented semi-automatically with HU of -250 to $-50^{37}$ using the same software (3D Slicer Chest Imaging Platform), and SFA was also measured if subcutaneous fat was fully covered. If metallic prosthesis was noted at umbilicus level, fat segmentation was not performed because of beam-hardening artifact. TFA was defined as a sum of VFA and SFA. All of segmentation was performed by one author (W.H.L., 6 years of experience in body imaging).

Collection of clinical information. Anthropometric data (height, body weight, WC), underlying medical diseases (hypertension, diabetes mellitus, dyslipidemia) and smoking status were collected by referring electronic medical records. If exact PY of smoking was available, PY was also recorded.

Statistical analysis. Normality of the numerical variables was assessed using Kolmogorov-Smirnov test. Numerical variables with normal distribution were provided with mean \pm standard deviation, while those not showing normal distribution were provided with median [interquartile range]. To compare multiple SMAs in chest CT with a reference standard (L3-SMA), linear regression was done. Annual change of each skeletal muscle area was calculated with individuals receiving multiple examinations. To explore the effect of scan parameters and reconstruction methods on SMA, multiple regression analysis was performed to adjust the age, sex, BMI, and VFA.

Lower 5th percentile values of SMI from young individuals (age $\leq 40$ years) ${ }^{21}$ were selected as Asian specific cut-offs for sarcopenia. Agreements of sarcopenia defined by L3-SMI and chest CT-derived sarcopenia were assessed by Cohen's Kappa coefficient $(\kappa)$. The $\kappa$ was interpreted as follows: (1) slight agreement $(\kappa, 0.01-0.20)$, (2) fair agreement $(\kappa, 0.21-0.40)$, (3) moderate agreement $(\kappa, 0.41-0.60)$, (4) substantial agreement $(\kappa, 0.61-0.80)$, and (5) almost perfect agreement $(\kappa, 0.81-0.99)^{38}$. Univariate logistic regression analysis and subsequent multivariate regression analysis with stepwise method were performed to explore factors related to sarcopenia. Factors with $p$-values less than 0.05 on univariate analysis were included on subsequent multivariate regression.

Age-dependent changes of SMAs were evaluated with linear regression and trends were visualized with locally weighted scattered-pot smoother (LOESS) with a span of $0.8^{39}$. To identify factors related with decrease of C/L1 ratio, linear and subsequent multiple regression analyses with stepwise method were applied.

Statistical analyses were performed using Medcalc (version 15.2, Ostend, Belgium). In this study, $p$-values less than 0.05 were considered statistically significant. Variance inflation factor (VIF) greater than 10 was considered as presence of multicolinearity, and variables with VIF greater than 10 were not included for the multivariate analysis.

\section{Data availability}

Data of our study population could be accessed as a separate excel file.

Received: 14 May 2021; Accepted: 23 December 2021

Published online: 10 January 2022

\section{References}

1. Cruz-Jentoft, A. J. et al. Sarcopenia: revised European consensus on definition and diagnosis. Age Ageing 48, 601. https://doi.org/ 10.1093/ageing/afz046 (2019).

2. Prado, C. M. et al. Prevalence and clinical implications of sarcopenic obesity in patients with solid tumours of the respiratory and gastrointestinal tracts: a population-based study. Lancet Oncol. 9, 629-635. https://doi.org/10.1016/S1470-2045(08)70153-0 (2008).

3. Su, H., Ruan, J., Chen, T., Lin, E. \& Shi, L. CT-assessed sarcopenia is a predictive factor for both long-term and short-term outcomes in gastrointestinal oncology patients: a systematic review and meta-analysis. Cancer Imaging 19, 82. https://doi.org/10. 1186/s40644-019-0270-0 (2019).

4. Portal, D. et al. L3 skeletal muscle index (L3SMI) is a surrogate marker of sarcopenia and frailty in non-small cell lung cancer patients. Cancer Manag. Res. 11, 2579-2588. https://doi.org/10.2147/CMAR.S195869 (2019). 
5. Choe, E. K., Lee, Y., Kang, H. Y., Choi, S. H. \& Kim, J. S. Association between CT-measured abdominal skeletal muscle mass and pulmonary function. J. Clin. Med. https://doi.org/10.3390/jcm8050667 (2019).

6. Okamura, H., Kimura, N., Mieno, M., Yuri, K. \& Yamaguchi, A. Preoperative sarcopenia is associated with late mortality after off-pump coronary artery bypass grafting. Eur. J. Cardiothorac. Surg. 58, 121-129. https://doi.org/10.1093/ejcts/ezz378 (2020).

7. Walston, J. D. Sarcopenia in older adults. Curr. Opin. Rheumatol. 24, 623-627. https://doi.org/10.1097/BOR.0b013e328358d59b (2012).

8. National Lung Screening Trial Research, T. et al. Reduced lung-cancer mortality with low-dose computed tomographic screening. N. Engl. J. Med. 365, 395-409, https://doi.org/10.1056/NEJMoa1102873 (2011).

9. Jin, G. Y. et al. Interstitial lung abnormalities in a CT lung cancer screening population: prevalence and progression rate. Radiology 268, 563-571. https://doi.org/10.1148/radiol.13120816 (2013).

10. de Torres, J. P. et al. Exploring the impact of screening with low-dose CT on lung cancer mortality in mild to moderate COPD patients: a pilot study. Respir. Med. 107, 702-707. https://doi.org/10.1016/j.rmed.2013.01.013 (2013).

11. Sanders, K. J. C., Degens, J., Dingemans, A. C. \& Schols, A. Cross-sectional and longitudinal assessment of muscle from regular chest computed tomography scans: L1 and pectoralis muscle compared to L3 as reference in non-small cell lung cancer. Int. J. Chron Obstruct. Pulmon Dis. 14, 781-789. https://doi.org/10.2147/COPD.S194003 (2019).

12. Recio-Boiles, A. et al. Enhancing evaluation of sarcopenia in patients with non-small cell lung cancer (NSCLC) by assessing skeletal muscle index (SMI) at the first lumbar (L1) level on routine chest computed tomography (CT). Support Care Cancer 26, 2353-2359. https://doi.org/10.1007/s00520-018-4051-2 (2018).

13. Kim, E. Y. et al. Evaluation of sarcopenia in small-cell lung cancer patients by routine chest CT. Support Care Cancer 24, 4721-4726. https://doi.org/10.1007/s00520-016-3321-0 (2016).

14. Tanimura, K. et al. Quantitative assessment of erector spinae muscles in patients with chronic obstructive pulmonary disease. Novel chest computed tomography-derived index for prognosis. Ann. Am. Thorac. Soc. 13, 334-341. https://doi.org/10.1513/Annal sATS.201507-446OC (2016).

15. Moon, S. W. et al. Thoracic skeletal muscle quantification: low muscle mass is related with worse prognosis in idiopathic pulmonary fibrosis patients. Respir. Res. 20, 35. https://doi.org/10.1186/s12931-019-1001-6 (2019).

16. Nakano, A. et al. Early decrease in erector spinae muscle area and future risk of mortality in idiopathic pulmonary fibrosis. Sci. Rep. 10, 2312. https://doi.org/10.1038/s41598-020-59100-5 (2020).

17. Rozenberg, D. et al. Thoracic muscle cross-sectional area is associated with hospital length of stay post lung transplantation: a retrospective cohort study. Transpl. Int. 30, 713-724. https://doi.org/10.1111/tri.12961 (2017).

18. McDonald, M. L. et al. Quantitative computed tomography measures of pectoralis muscle area and disease severity in chronic obstructive pulmonary disease. A cross-sectional study. Ann. Am. Thorac. Soc. 11, 326-334. https://doi.org/10.1513/AnnalsATS. 201307-229OC (2014).

19. Magudia, K. et al. Population-scale CT-based body composition analysis of a large outpatient population using deep learning to derive age-, sex-, and race-specific reference curves. Radiology 298, 319-329. https://doi.org/10.1148/radiol.2020201640 (2021).

20. Cho, Y. H. et al. Association of chest CT-based quantitative measures of muscle and fat with post-lung transplant survival and morbidity: a single institutional retrospective cohort study in Korean population. Korean J. Radiol. 20, 522-530. https://doi.org/ 10.3348/kjr.2018.0241 (2019).

21. Derstine, B. A. et al. Skeletal muscle cutoff values for sarcopenia diagnosis using T10 to L5 measurements in a healthy US population. Sci. Rep. 8, 11369. https://doi.org/10.1038/s41598-018-29825-5 (2018).

22. Guerri, R. et al. Mass of intercostal muscles associates with risk of multiple exacerbations in COPD. Respir. Med. 104, 378-388. https://doi.org/10.1016/j.rmed.2009.10.015 (2010).

23. McLean, R. R. et al. Criteria for clinically relevant weakness and low lean mass and their longitudinal association with incident mobility impairment and mortality: the foundation for the National Institutes of Health (FNIH) sarcopenia project. J. Gerontol. A Biol. Sci. Med. Sci. 69, 576-583. https://doi.org/10.1093/gerona/glu012 (2014).

24. Weaver, A. A., Schoell, S. L. \& Stitzel, J. D. Morphometric analysis of variation in the ribs with age and sex. J. Anat. 225, 246-261. https://doi.org/10.1111/joa.12203 (2014).

25. Diaz, A. A. et al. Pectoralis muscle area and mortality in smokers without airflow obstruction. Respir. Res. 19, 62. https://doi.org/ 10.1186/s12931-018-0771-6 (2018).

26. Degens, H., Gayan-Ramirez, G. \& van Hees, H. W. Smoking-induced skeletal muscle dysfunction: from evidence to mechanisms. Am. J. Respir. Crit. Care Med. 191, 620-625. https://doi.org/10.1164/rccm.201410-1830PP (2015).

27. Gea, J., Pascual, S., Casadevall, C., Orozco-Levi, M. \& Barreiro, E. Muscle dysfunction in chronic obstructive pulmonary disease: update on causes and biological findings. J. Thorac. Dis. 7, E418-438. https://doi.org/10.3978/j.issn.2072-1439.2015.08.04 (2015).

28. Kim, D. W. et al. Reliability of skeletal muscle area measurement on CT with different parameters: a phantom study. Korean J. Radiol. 22, 624-633. https://doi.org/10.3348/kjr.2020.0914 (2021).

29. Magudia, K. et al. Population-scale CT-based body composition analysis of a large outpatient population using deep learning to derive age-, sex-, and race-specific reference curves. Radiology https://doi.org/10.1148/radiol.2020201640 (2020).

30. Graffy, P. M. et al. Deep learning-based muscle segmentation and quantification at abdominal CT: application to a longitudinal adult screening cohort for sarcopenia assessment. Br. J. Radiol. 92, 20190327. https://doi.org/10.1259/bjr.20190327 (2019).

31. Park, H. J. et al. Development and validation of a deep learning system for segmentation of abdominal muscle and fat on computed tomography. Korean J. Radiol. 21, 88-100. https://doi.org/10.3348/kjr.2019.0470 (2020).

32. Yu, S. et al. Robustness study of noisy annotation in deep learning based medical image segmentation. Phys. Med. Biol. $65,175007$. https://doi.org/10.1088/1361-6560/ab99e5 (2020).

33. Edwards, K. et al. Abdominal muscle segmentation from CT using a convolutional neural network. Proc. SPIE Int. Soc. Opt. Eng. https://doi.org/10.1117/12.2549406 (2020).

34. van Vugt, J. L. A. et al. Contrast-enhancement influences skeletal muscle density, but not skeletal muscle mass, measurements on computed tomography. Clin. Nutr. 37, 1707-1714. https://doi.org/10.1016/j.clnu.2017.07.007 (2018).

35. Chang, Y. et al. Alcoholic and non-alcoholic fatty liver disease and associations with coronary artery calcification: evidence from the Kangbuk Samsung Health Study. Gut 68, 1667-1675. https://doi.org/10.1136/gutjnl-2018-317666 (2019).

36. Lee, C. et al. Health and Prevention Enhancement (H-PEACE): a retrospective, population-based cohort study conducted at the Seoul National University Hospital Gangnam Center, Korea. BMJ Open 8, e019327. https://doi.org/10.1136/bmjopen-2017-019327 (2018).

37. Lee, A. et al. Cut-off values for visceral fat area identifying korean adults at risk for metabolic syndrome. Korean J. Fam. Med. 39, 239-246. https://doi.org/10.4082/kjfm.17.0099 (2018).

38. Viera, A. J. \& Garrett, J. M. Understanding interobserver agreement: the kappa statistic. Fam. Med. 37, 360-363 (2005).

39. Zhang, Z., Zhang, H. \& Khanal, M. K. Development of scoring system for risk stratification in clinical medicine: a step-by-step tutorial. Ann. Transl. Med. 5, 436. https://doi.org/10.21037/atm.2017.08.22 (2017).

\section{Author contributions}

W.H.L. and C.M.P. designed the study, wrote the main manuscript, and reviewed the main manuscript. W.H.L. collected, analyzed and interpreted the data. 


\section{Competing interests}

The authors declare no competing interests.

\section{Additional information}

Supplementary Information The online version contains supplementary material available at https://doi.org/ 10.1038/s41598-021-04492-1.

Correspondence and requests for materials should be addressed to C.M.P.

Reprints and permissions information is available at www.nature.com/reprints.

Publisher's note Springer Nature remains neutral with regard to jurisdictional claims in published maps and institutional affiliations.

(c) (1) Open Access This article is licensed under a Creative Commons Attribution 4.0 International License, which permits use, sharing, adaptation, distribution and reproduction in any medium or format, as long as you give appropriate credit to the original author(s) and the source, provide a link to the Creative Commons licence, and indicate if changes were made. The images or other third party material in this article are included in the article's Creative Commons licence, unless indicated otherwise in a credit line to the material. If material is not included in the article's Creative Commons licence and your intended use is not permitted by statutory regulation or exceeds the permitted use, you will need to obtain permission directly from the copyright holder. To view a copy of this licence, visit http://creativecommons.org/licenses/by/4.0/.

(c) The Author(s) 2022 